\title{
Use of regulators and inhibitors of Pim-1, a serine/threonine kinase, for tumour therapy (Review)
}

\author{
CHEN LIANG and YING-YI LI \\ Department of Oncology, Cancer Research Institute, Fudan University Shanghai Cancer Center, \\ Shanghai Medical College, Fudan University, Shanghai 200032, P.R. China
}

Received August 31,2013; Accepted March 11, 2014

DOI: $10.3892 / \mathrm{mmr} .2014 .2139$

\begin{abstract}
Pim-1 is a proto-oncogene that encodes a serine/threonine kinase that is overexpressed in a range of haematopoietic malignancies and solid cancers. Pim-1 expression is tightly regulated by multiple biomolecules at different levels. Several lines of evidence have indicated that dysregulation of Pim-1 can interfere with the cell cycle and apoptosis to promote malignant transformation of a number of types of tumour. Thus, investigation of Pim-1 regulation may provide important theoretical guidance for the development of molecular targeting therapies and drug treatments for Pim-1-associated diseases. Regulators of Pim-1 expression, include microRNAs, oestrogen, inecalcitol, adenosine triphosphate (ATP) mimetic inhibitors and ATP competitive inhibitors of Pim-1. Combinations of inhibitors of Pim-1 expression and Pim-1-specific inhibitors may provide novel therapies for cancer patients and directions for cancer treatment.
\end{abstract}

\section{Contents}

1. Introduction

2. Structure and biological functions of Pim-1

3. Regulators of Pim-1 expression

4. Pim-1 inhibitors

5. Conclusion

\section{Introduction}

Pim was initially identified by cloning the retroviral integration sites in murine Moloney leukaemia virus

Correspondence to: Professor Ying-Yi Li, Cancer Research Institute, Fudan University Shanghai Cancer Center, 270 DongAn Road, Shanghai 200032, P.R. China

E-mail: 12211230007@fudan.edu.cn

Key words: Pim kinase, microRNA, hormone, phosphoinositide 3-kinase-like protein kinase, cytokine, inhibitor
(MMLV)-induced lymphomas (1). Pim is a member of the family of oncoproteins that exhibit serine/threonine kinase activity (1). Furthermore, all Pim proteins were confirmed to contain an active site, termed the adenosine triphosphate (ATP) anchor. Pim genes represent a family of proto-oncogenes that encode three different kinases (Pim-1, -2 and -3) belonging to the $\mathrm{Ca}^{2+} /$ calmodulin-dependent protein kinase group, essential in the regulation of signal transduction cascades (2). Pim kinases are evolutionarily conserved, exhibiting a high degree of homology in sequence and structure (3). Pim kinases are normally constitutively active and are broadly expressed in haematopoietic, vascular smooth muscle and epithelial cell lineages, as well as in embryonic stem cells. Thus, they are essential for the normal growth and maturation of these cells.

MMLV proviral insertion in the 3'-untranslated region (UTR) of Pim-1 led to an increase in the stability of Pim-1 mRNA and Pim-1 protein expression, resulting in tumourigenesis of the T-cell lymphoma (4). Moreover, Pim-1 can prevent apoptosis and promote cell proliferation, effects which are considered important for malignant transformation (5). Furthermore, elevated Pim-1 expression levels have been observed in human haematopoietic malignancies as well as in solid tumours (6-8).

Under physiological circumstances, the body tightly controls the expression of the genes responsible for regulation of cell growth, apoptosis and the cell cycle. However, in a number of pathological conditions, dysregulation of these genes can lead to cellular dysplasia and induce malignant transformation of cells. Pim-1 is an oncogene that is important in the regulation of cell growth. The crystal structure of Pim-1 reveals the absence of an identified regulatory domain, which indicates that it does not depend on post-transcriptional modifications for activation and thus is constitutively active. Therefore, regulation of Pim-1 kinase activity largely depends on its protein expression level rather than its phosphorylation level (9). Notably, Pim-1 expression is not only transcriptionally mediated by a number of molecules but is also transiently induced by microRNAs and hormones at the post-transcriptional level. The mechanisms of cell gene expression regulation are diverse (10). In the present review, the regulation of Pim- 1 expression and a number of its inhibitors are discussed, providing theoretical guidance for the development of molecular targeting therapies and drug treatments for Pim-1-associated diseases. 


\section{Structure and biological functions of Pim-1}

In humans, the Pim-1 oncogene, $\sim 5 \mathrm{~kb}$ in length, is located on the short arm of chromosome 6p21.1-p21.31. The mRNA transcript for Pim-1 is encoded by six exons with large 5'- and 3'UTRs containing a $\mathrm{G} / \mathrm{C}$-rich region and five copies of AUUA destabilising motifs (2). Pim-1 can generate two isoforms (34 and $44 \mathrm{kD}$ ) due to alternative translation initiation points at an upstream CUG codon. The shorter form localises to the cytoplasm and the nucleus, whereas the longer form localises to the plasma membrane; however, the two proteins retain their serine/threonine kinase activity. The oncogenic activity of Pim kinases is mediated by multiple cellular substrates (6). Pim-1 kinase adopts a two-lobed kinase fold structure with a deep cleft between the $\mathrm{N}$ - and $\mathrm{C}$-terminal lobes connected via the hinge region (residues 121-126). The $\mathrm{N}$-terminal lobe is composed primarily of $\beta$-sheets, whereas the $\mathrm{C}$-terminal lobe is comprised of $\alpha$-helices. The hinge region has specific residues identified as ATP-binding sites. Moreover, the ATP-binding pocket in Pim-1 is always open, indicating that Pim-1 kinase constitutively resides in an active conformation $(9,11,12)$.

The Pim- 1 oncogene is frequently overexpressed in a range of haematological malignancies and several solid tumours. Its activity supports tumour cell growth and survival in vitro and in vivo through phosphorylation of a large number of common substrates, including several cell cycle regulators and apoptosis mediators. Pim-1 kinase can phosphorylate Cdc25A (G1/S), Cdc25C (G2/M), p21 $1^{\mathrm{CIP} 1 / \mathrm{WAF} 1}$ and $\mathrm{p} 27$, all of which are involved in the regulation of the cell cycle $(7,13-16)$. It is also able to inactivate Bad protein by phosphorylating ser ${ }^{112}$, impeding the process of apoptosis and supporting tumour cell growth and survival. Additionally, it interacts with the nuclear mitotic apparatus protein to promote mitosis (2). Thus, Pim-1 may alter various biological functions to accelerate oncogenesis by inhibiting apoptosis, enhancing cell proliferation and promoting cell differentiation.

\section{Regulators of Pim-1 expression}

Regulation at the transcriptional level. Similar to other protein kinases, Pim-1 expression is known to be regulated mainly by the rate of transcription. Binding of a wide range of growth factors, hormones and cytokines, such as interleukins, epidermal growth factor, prolactin, granulocyte colony-stimulating factor and granulocyte-macrophage colony-stimulating factor, to target surface-specific receptors activates the Janus kinase/signal transducer and activator of transcription (JAK/STAT) signal transduction pathway, which is essential for regulating Pim-1 gene expression. Janus kinases subsequently phosphorylate the cytoplasmic receptor tyrosine kinase domain, thus generating recruitment sites for STATs containing the SH2 domain. The activation of STATs phosphorylated by JAK leads to their dimerisation and nuclear translocation. In the nucleus, STAT3 and STAT5 directly bind to the Pim-1 promoter at the ISFR/GAS sequence, thus upregulating the transcription of Pim-1. In addition, Pim-1 itself is able to negatively regulate the JAK/STAT pathway by binding to a group of negative regulators, termed suppressor of cytokine signalling proteins (2). In BCR/ABL-expressing oncoprotein cells, Pim-1 is markedly upregulated following activation of BCR-ABL tyrosine kinase by activation of STAT5 (17). Moreover, nuclear factor- $\kappa \mathrm{B}$ can rapidly induce Pim-1 expression following tumour necrosis factor- $\alpha$ stimuli in a STAT5-mediated manner (18). Thus, multiple signalling pathways have STATs at their core, forming a complex network to co-regulate Pim-1 expression at the transcriptional level (Fig. 1).

Regulation via knockdown of Pim-1 expression using microRNA. MicroRNAs (miRNAs) are a family of small noncoding RNA molecules, 22 nucleotides (nt) in length, which post-transcriptionally silence target gene expression to act as either oncogenes or tumour-suppressor genes (19-21). Several miRNAs have been identified to be associated with Pim-1-related tumour initiation. Downregulation of Pim-1 by miRNAs, which may contribute to the differential expression of Pim-1 in tumours versus normal cells, regulates the cell cycle and apoptosis. Analysis of the structure of human Pim-1 mRNA indicates that the 3'UTR of Pim-1, which is generally evolutionarily conserved, harbours multiple binding sites for miRNAs (22). Additionally, the 'seed region' covering nucleotides 2-8 of the mature miRNA strand is essential for interacting with the target miRNA to destabilise it and inhibit its translation $(23,24)$ (Fig. 1).

miRNA-16 acts as an important tumour suppressor by regulating pro-oncogene expression (25). Various types of cancer cells, including chronic lymphocytic leukaemia (CLL) and prostate cancer cells, have been shown to reduce miRNA-16 levels $(26,27)$. Fms-like tyrosine kinase 3 (FLT3), is expressed in a large number of acute myeloid leukaemia (AML) cases, and is activated by internal tandem duplication (ITD) mutations, which aberrantly activate its downstream signalling to contribute to cell proliferation and inhibit apoptosis (28). This may explain why AML patients with the FLT3/ITD mutant phenotype have a poor clinical prognosis (28-31). Notably, in FLT3/ITD-expressing cells, Pim-1 is upregulated and is involved in FLT3-mediated cell survival $(32,33)$, whereas miRNA-16 is downregulated. Furthermore, based on bioinformatics analysis, miRNA-16 may target the 3'UTR of human Pim-1 (22,34). Using quantitative polymerase chain reaction and immunoblotting, Pim-1 mRNA and protein levels were confirmed to be markedly decreased upon miRNA-16 mimic transfection in FLT3/ITD-expressing cells. Therefore, miRNA-16 appears to bind to the 3'UTR putative target site of Pim-1 and directly inhibit its expression at the post-transcriptional level, thus slowing down the growth of FLT3/ITD-expressing cells (34). However, enforced miRNA-16 expression could not completely deplete Pim-1 expression in FLT3/ITD-expressing cells, suggesting continuous induction of Pim-1 by other FLT3/ITD-mediated signalling molecules (34). Activation of FLT3 can also activate numerous signal transduction pathways, including STAT5 phosphorylation (35-37), for the upregulation of Pim-1 (38). Thus, Pim-1 may be a predominant downstream target of multiple signalling pathways activated by FLT3. Notably, miRNA-16 expression is comparably high in the K562 myeloid leukaemia cell line and the LS174T colon cancer cell line, but miRNA-16 did not affect Pim-1 expression in either cell line (22). In conclusion, FLT3/ITD and Pim-1 are important in the transformation of leukaemia cells. This evidence supports 


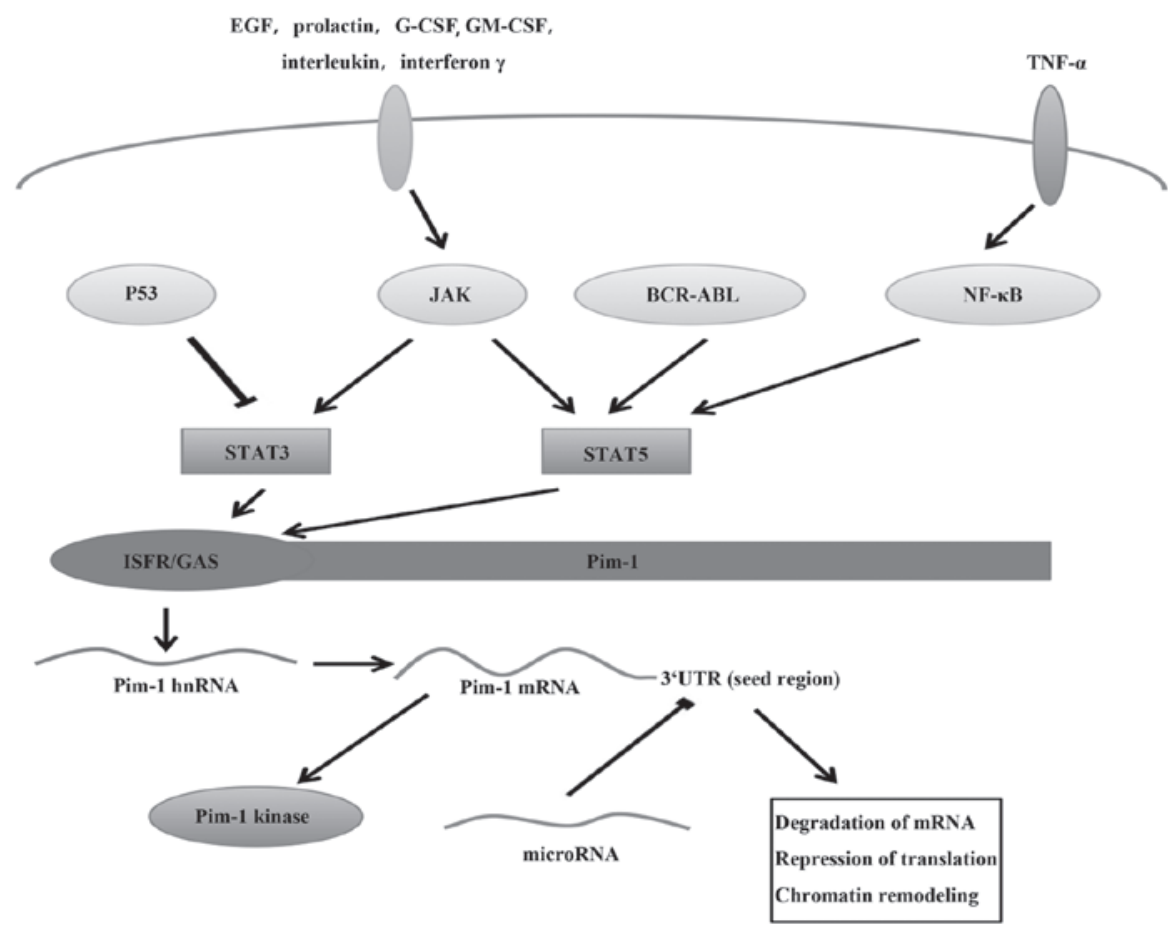

Figure 1. Regulation of Pim-1 at the transcriptional level via the JAK/STAT signalling pathway and at the post-transcriptional level via miRNA. Receptor stimulation by hormones, cytokines and growth factors, such as interleukins, interferon $\gamma$, EGF, prolactin, G-CSF and GM-CSF, activate STAT by JAKs, BCR-ABL and NF-кB. p53 can inhibit STAT3. STAT3 and STAT5 upregulate the transcription of Pim-1 by binding to the Pim1 promoter at the ISFR/GAS-sequence, since STAT proteins serve as transcription factors for Pim genes following engagement of their cognate ligands. miRNAs can bind to the seed region in the Pim-1 3'UTR, inhibiting Pim-1 expression to result in mRNA destabilisation and translational inhibition. JAK, Janus kinase; STAT, signal transducer and activator of transcription; miRNA, microRNA; EGF, epidermal growth factor; G-CSF, granulocyte colony-stimulating factor; GM-CSF, granulocyte-monocyte colony-stiumulating factor; 3'UTR, 3'-untranslated region; TNF, tumour necrosis factor; hnRNA, heterogenous nuclear RNA.

the hypothesis that FLT3 should be routinely analysed to guide therapy and estimate prognosis in AML patients, and may be used as a novel target for chemotherapeutics to treat patients with leukaemia. Moreover, miRNA-16 is an important component of the FLT3/ITD signalling pathway, and it may serve as a clinically useful biomarker and permit the development of novel drugs.

miRNA-33 has two isoforms, miRNA-33a and miRNA-33b, involved in the regulation of gene transcription associated with cholesterol biosynthesis and uptake (39), but their relevance with regard to tumours has rarely been explored. Notably, in a variety of cancer cell lines, miRNA-33a expression has been revealed to be generally low, with Pim- 1 expression relatively high. miRNA-33a significantly reduces Pim-1 expression in K562 and LS174T cells via specifically binding to the seed region of Pim-1. The repression effects were essentially abrogated in the presence of a mutated seed region (22). However, miRNA-33a was not shown to affect Pim-1 expression in FLT3/ITD expressing cells, thus the regulation is cell-specific (34). In MM.1S multiple myeloma cells, Pim-1 is also a direct target of miRNA-33b, participating in the apoptosis induced by miRNA-33b (40). However, miRNA-33b is not considered to be the primary miRNA to regulate Pim-1 in K562 and LS174T cells, although levels of miR-33b are observed to be low in the two cell lines (22).

Ibrahim et al (41) preclinically validated a polyethylenimine (PEI)-mediated method of unmodified miRNA-33a delivery in a mouse model of colon carcinoma through in vitro and in vivo experiments. This method can efficiently strengthen the repression of Pim-1 expression in vivo in tumour cells, and its antitumour effect is similar to that of Pim-1 knockdown using small interfering (si)RNA/PEI. Moreover, the inhibition effect of the modified miRNA-33a mimic resembles that of siRNAs fully complementary to the miRNA-33a target site, but is more efficient than the unmodified mimic (22). Thus, this approach using miRNAs for cancer therapy is expected to become an efficient and biocompatible strategy for targeting Pim-1.

miRNA-1 expression is highest in various types of muscle cell, including spindle-shaped and vascular smooth muscle cells, mediating cell proliferation and differentiation in cardiac and skeletal muscle cells $(42,43)$. Notably, Pim- 1 is able to promote proliferation of cultured smooth muscle cells (SMCs) and neointimal hyperplasia in vitro (44). Chen et al (45) demonstrated that miRNA-1 is a downstream effector of myocardin and inhibits the proliferation of SMCs mediated by myocardin. Furthermore, miRNA-1 may mediate the repression of Pim-1 expression at the translational level to inhibit vascular SMC proliferation. In conclusion, miRNA-1 is able to inhibit SMC proliferation and may be a novel target site for vascular smooth muscle proliferative diseases.

Diabetic cardiomyopathy was characterised as having reduced Pim-1 levels in the early stages of the disease, along with reduced upstream activators, p-STAT3 and p-Akt (46). Notably, in a model of diabetic cardiomyopathy, miRNA-1 was upregulated from an early phase and was positively correlated with the progression of diabetic cardiomyopathy, indicating that miRNA-1 can negatively modulate Pim-1 to mediate the 


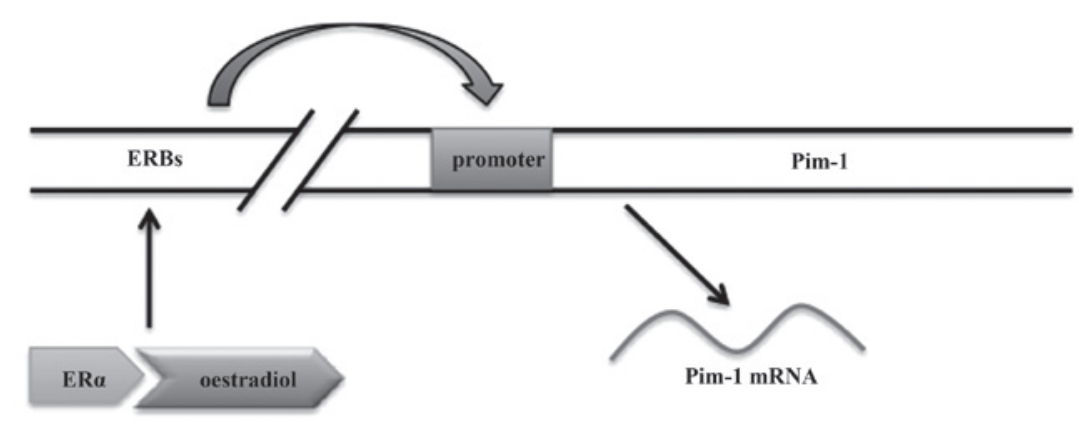

Figure 2. Regulation of Pim-1 expression by oestradiol. The oestradiol-loaded ER- $\alpha$ binding to ERBs, far upstream of the Pim-1 promoter, potentially enhances promoter activation via the interaction of various ERBs. Subsequently, Pim-1 expression is upregulated. ER- $\alpha$, oestradiol receptor $\alpha$; ERB, ER- $\alpha$ binding site.

further progression of cardiomyopathy (47). Katare et al (47) performed in vitro cell experiments demonstrating that miRNA-1 not only inhibited Pim-1 directly but also via its upstream modulator, Akt. Moreover, anti-miRNA-1 could restore Pim-1 expression and the proliferative activity of cardiac progenitor cells. Thus, an imbalance between negative and positive regulators leads to the repression of Pim-1 with advancing cardiomyopathy. This provides insight into novel strategies for gene therapy for this disease.

Although miRNA-1 levels in other tissues are lower than in the muscle (48), comparison of genomic positions of mouse tumour susceptibility loci with those of mouse miRNA genes revealed that the flanking region of miRNA-1 has six substitutions affecting susceptibility to lung cancer (49). It has previously been reported that in human primary lung cancer tissues and almost all lung cancer cell lines, miRNA-1 expression is comparably low, whereas Pim-1 expression is significantly upregulated $(12,50,51)$. A study has revealed that miRNA-1 binding to the 3'UTR of Pim-1 negatively affects regulation of the antitumour effect of Pim-1 (50). Thus, the inhibitory mechanism of Pim-1 expression mediated by miRNA-1 exists not only in muscle cells, but also in tumour cells. As miRNA-1 has diverse roles in various diseases, due to the wide distribution in levels of miRNA-1 expression, further studies into its regulation of tumour-associated angiogenesis are warranted.

Several studies have determined that Pim-1 kinase is important in the survival of $\mathrm{BCR} / \mathrm{ABL}^{+}$cells (52). miRNA-328 expression is downregulated, whereas Pim-1 protein is markedly upregulated in $\mathrm{BCR} / \mathrm{ABL}^{+}$cells. One study has demonstrated that miRNA-328 interacting with the $3^{\prime}$ UTR of Pim-1 could inhibit Pim-1 expression, blocking cell proliferation and growth (53).

miRNA-210 is inducible by hypoxia and appears to be a hypoxia-inducible factor target gene $(54,55)$. Huang et al $(56)$ identified Pim-1 as an miRNA-210 target gene through the miRNP-IP approach followed by cloning the 3'UTR of Pim-1 to perform reporter assays. However, luciferase activity was repressed by $15 \%$ in the Pim-1 construct compared with co-transfection with a control plasmid. In addition, the inhibitory effect of miRNA-210 on tumour growth initiation was not rescued by expressing the Pim-1 coding sequence without the 3'UTR. These observations suggest that Pim-1 may be a weak miRNA-210 target gene, although Pim-1 is enriched by microarray analysis.
In conclusion, the expression of various miRNAs depends on the pathological cell type and multiple stimuli. miRNA can simultaneously regulate multiple target genes and Pim-1 is regulated by multiple miRNAs. Notably, different primary miRNAs regulate Pim-1 expression in different tissues and cells. Thus, in tumour cells with Pim-1 overexpression, identifying the miRNAs important in regulating Pim-1 expression requires further research. Moreover, the 3'UTR of Pim-1 harbours numerous other miRNA binding sites, that may be important in the development of various diseases.

Regulation of Pim-1 expression by hormones. Previous studies have shown that one of the major risk factors of breast cancer is cumulative oestrogen exposure (57). Oestrogen receptor $\alpha$ (ER- $\alpha$ ) inhibits Forkhead box protein M1 expression, which is involved in the occurrence and development of breast cancer induced by oestrogen (58). It has been recently reported that there are four binding sites for oestradiol (E2)-loaded ER- $\alpha$ far upstream of the Pim- 1 promoter, and these ER- $\alpha$-binding regions (ERBs) may function as oestrogen-regulated enhancers for Pim-1 (59). Oestradiol rapidly triggers loading of ER- $\alpha$ to the ERBs, then ERBs interact with each other via chromatin loop formation, resulting in Pim-1 expression. Thus, Pim-1 is a direct ER- $\alpha$ target in breast cancer cells and oestradiol positively regulates its expression in an ER- $\alpha$-mediated manner (Fig. 2). Furthermore, Pim-1 overexpression induced by oestradiol has been determined to phosphorylate and thereby inhibit the expression of cell cycle inhibitors, hindering apoptosis, promoting cell cycle progression and increasing invasiveness of breast cancer tumours. Collectively, these results add a novel potential mechanism by which oestradiol is able to promote breast cancer cell proliferation (59).

Dehydroepiandrosterone (DHEA) is an abundantly produced steroid hormone, known to improve pulmonary arterial hypertension $(\mathrm{PAH})$ through its vasodilator properties and reverse vascular remodelling (60). Paulin et al (61) investigated pulmonary artery smooth muscle cells (PASMCs) in PAH, and demonstrated a significant decrease in the p-STAT3/STAT3 ratio and the nuclear translocation of p-STAT3 following treatment of the PAH-PASMCs with DHEA. Similarly, DHEA also decreases Pim-1 mRNA and protein levels in PAH-PASMCs. Since Pim-1 is the STAT3 downstream target implicated in $\mathrm{PAH}$, the results suggest that DHEA can downregulate Pim-1 expression via decreasing the quantity of phosphorylated STAT3 for activation in PAH-PASMCs. 


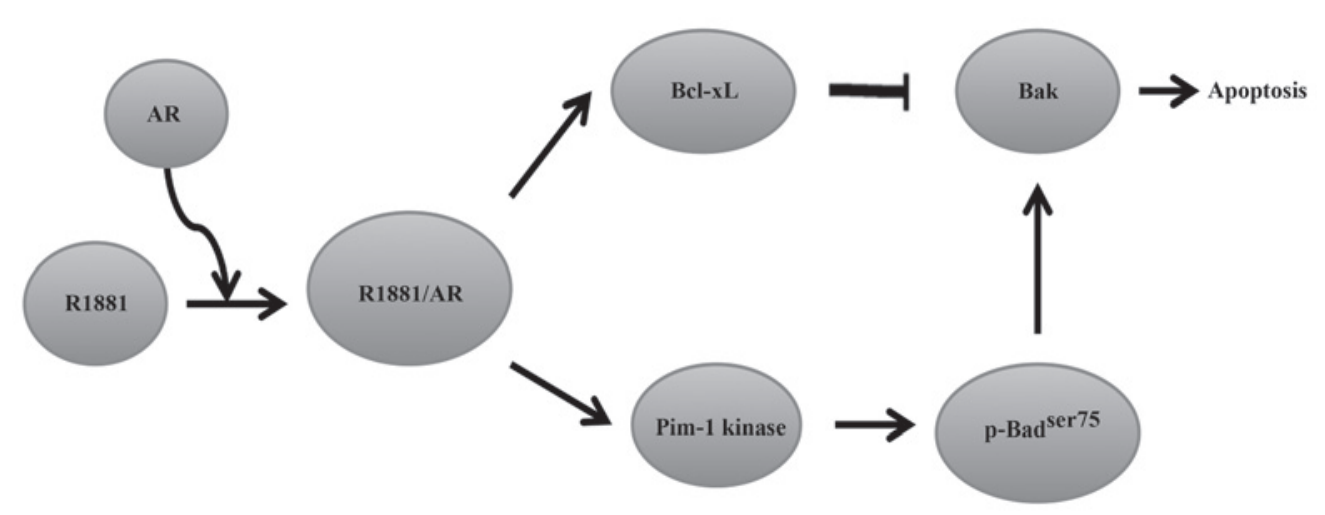

Figure 3. The pro-survival pathways activated by R1881 in LNCaP prostate cancer cells. The R1881-loaded AR induces an increase in Bcl-xL expression, which sequesters Bak at the mitochondria, preventing its activation to exert anti-apoptotic effects. The R1881-loaded AR can also phosphorylate Bad by increasing the Pim-1 kinase activity to efficiently enhance the survival advantage of the AR-mediated increase in Bcl-xL expression by preventing displacement of Bak from Bcl-xL. AR, androgen.

Pim-1 overexpression in prostate cancer cells has been associated with tumourigenesis (62). Preclinical data regarding Vitamin D3 (calcitriol) have revealed antiproliferative and apoptosis-inducing effects resulting in significant antitumour activities in prostate cancer cells $(62,63)$. Since calcitriol treatment can result in hypercalcaemia, the dose that can be administered to patients is less than that theoretically required for antitumour activity. Consequently, Okamoto et al (64) synthesised and tested inecalcitol, a novel and unique analogue of vitamin D3 that is potent but less calcaemic. Pim-1 expression was shown to be decreased in a dose-dependent manner following treatment of $\mathrm{LNCaP}$ prostate cancer cells and LNCaP xenografts with inecalcitol or calcitriol, respectively. In addition, inecalcitol was more potent than calcitriol in downregulating the levels of Pim-1 mRNA and protein.

It is reported that Pim-1 overexpression can downregulate the androgen receptor (AR)-mediated signalling that inhibits cell proliferation and induces dedifferentiation by AR phosphorylation (65). Notably, Pim-1 is closely associated with hormone refractory prostate cancers (65). Therefore, administration of inecalcitol may have a positive impact on the therapy of androgen-dependent prostate cancer.

Conversely, Maier et al (66) identified that Pim-1 kinase may not be a calcitriol target gene in $\mathrm{HaCaT}$ keratinocytes. However, Pim-1 can interact with the vitamin D3 receptor (VDR) DNA-binding domain, participating in signal transduction of calcitriol. Further research into the mechanism involved in the interaction of Pim-1 and calcitriol is required.

Androgens are the key male hormones involved in the development of the prostate gland. Androgens can promote the development of androgen-dependent prostate cancer mediated through the androgen receptor, which is a key hormone correlated with prostate cancer, and androgen deprivation therapy is a common treatment for prostate cancer patients $(67,68)$. The phosphatidylinositol 3-kinase (PI3K) inhibitor LY294002 can induce apoptosis in serum-deprived LNCaP prostate cancer cells. The apoptosis-inducing effect is significantly neutralised by androgen methyltrienolone, R1881, resulting in cell survival and proliferation (69). Moreover, the prosurvival effects of R1881 are linked to Bcl-xL overexpression (70). Kumar et al (71) demonstrated that activation of AR by R1881 induced an increase in Bcl-xL expression, which contributed to sequestering the pro-apoptotic protein Bak, thereby preventing its activation and the accompanying prosurvival effects. In addition, the authors confirmed that the pro-survival effect of $\mathrm{Bcl}-\mathrm{xL}$ requires an increase in the stability of protein kinase Pim-1. Furthermore, the results indicated that the increase of Pim-1 kinase activity and stability correlated with an increase in the half-life of Pim-1 by R1881 induction rather than an increase in the transcription rate. Notably, R1881-induction was not caused by an increase in the quantity of Pim-1 protein. The enhanced activity of Pim-1 kinase prevented full activation of Bad via phosphorylation of Bad at $\mathrm{ser}^{75}$ and offset dephosphorylation of Bad by LY294002, and enhanced Bcl-xL to exert its anti-apoptotic activity through the sequestration of Bak (Fig. 3) (71). In conclusion, these results have improved the understanding of the molecular mechanism of tumourigenesis of prostate cancer to provide novel insights for the treatment of androgen-dependent prostate cancer.

The emerging identification of the importance of hormone imbalance in the development of human tumours has increased interest in the development of hormone-associated drugs. As more is revealed concerning the hormone-associated mechanisms of Pim- 1 expression, more will be understood about the association between hormones and tumour development. Targeting Pim-1 may offer a strategy for improved treatment of hormone-dependent tumours.

Regulation of Pim-1 expression by PI3K-like kinases. DNA-dependent protein kinase complex (DNA-PK) and Ataxia-Telangiectasia Mutated (ATM) are members of the PI3K-like kinase family. Akt is a downstream effector of PI3K and LY294002 is an inhibitor of PI3K-like kinases. The expression of Pim-1, Pim-2 and Pim-3 mRNA is rapidly increased following treatment of endothelial cells (ECs) with LY294002, but there is no effect on the stability of the mRNA, indicating that LY294002 can regulate the activity of the promoters of Pim to induce the upregulation of Pim expression (72). Akt overexpression has been reported to increase Pim-1 expression in neonatal rat cardiomyocytes (73). Similarly, Pim-1 is a crucial downstream target of Akt in ECs, and Akt can increase Pim-1 expression, but does not affect Pim-2 and Pim-3 expression. In addition, DNA-PK and ATM can decrease Pim- 1 expression in physiological conditions (72). Thus, PI3K-like 


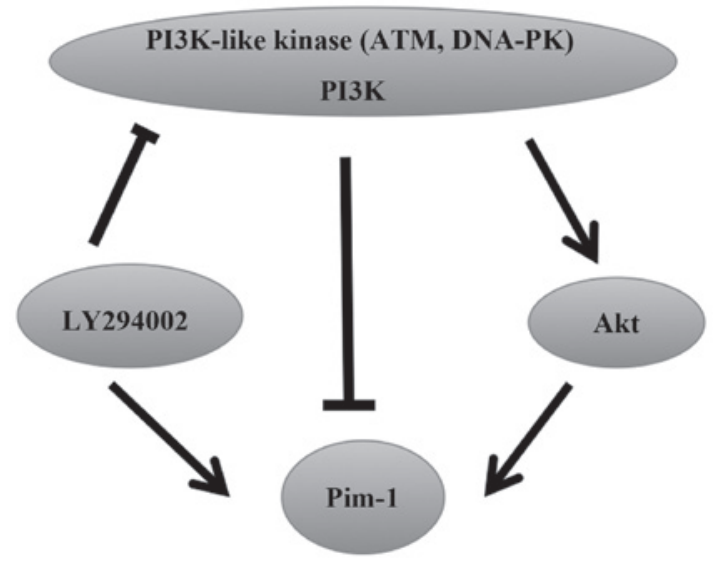

Figure 4. PI3K-like kinases can simultaneously positively and negatively regulate Pim-1 expression. PI3K-like kinases not only induce Akt overexpression to subsequently upregulate Pim-1 expression, but also directly inhibit the expression of Pim-1. LY294002, an inhibitor of the PI3K-like kinases, also increases Pim-1 expression. PI3K, phosphatidylinositol 3-kinase; ATM, ataxia telangiactasia mutated; DNA-PK, DNA-dependent protein kinase.

kinases have dual effects on the regulation of Pim expression (Fig. 4).

Regulation of Pim-1 expression by cytokines. The vascular endothelial growth factor (VEGF)-A/Flk-1 signalling pathway also increases the Pim-1 expression level. Zippo et al (74) identified Pim-1 as a target gene of Flk-1, which is induced during the process of angiogenesis. Furthermore, in angiogenesis of human umbilical cord vein endothelial cells, VEGF-A can induce Pim-1 expression mediated by Flk-1, although this induction is poor with Pim-1 expression levels only marginally increased. Consistent with this finding, this mechanism also exists in vivo in ECs during angiogenesis of the ovary (74). However, platelet-derived growth factor bb (PDGFbb), but not VEGF-A165, can transcriptionally stimulate Pim-1 expression in vascular smooth muscle cells (VSMC), mostly attributable to the activation of JAK/STAT, but also to an additional pathway involving protein kinase $\mathrm{C}(\mathrm{PKC})$ and the mitogen-activated protein kinase Mek1/2, leading to the expression of the Pim-1 kinase and proliferation of VSMCs $(75,76)$. Moreover, it has been recently reported that interleukin (IL)-6 stimulates STAT3 and Pim-1 kinase in pancreatic cancer cell lines and that the increase in IL-6-stimulated Pim-1 may be partially STAT3-independent (77).

\section{Pim-1 inhibitors}

ATP mimetic inhibitors. ATP mimetic inhibitors bound to Pim-1 are sandwiched between hydrophobic residues from the glycine-rich loop, the C-terminal domain of Pim-1 kinase and the hinge region. The presence of proline at position 123 prevents the molecules from forming the second hydrogen bond to the hinge, thereby only one hydrogen bond between the ligand and the hinge is observed (78). These inhibitors comprise the broad-spectrum kinase inhibitor staurosporine and its analogue K252, bisindoylmaleinimides and the related PKC inhibitor LY333531, as well as a number of extremely potent organometallic inhibitors (18). Medical research into this class of inhibitors has thus far been limited.

ATP competitive inhibitors. ATP competitive inhibitors do not interact with the hinge region by forming classical hydrogen bonds so can therefore be considered as ATP competitive inhibitors and not ATP mimetic inhibitors. SGI-1776, SMI-4a, LY294002, quercetagetin, 1,10-dihydropyrrolo[2,3- $\alpha$ ] carbazole-3-carbaldehyde (DHPCC-9) and more recently, pyrrolo[2,3-g]indazoles have been idenfied as ATP competitive inhibitors(79). A number of these compounds are in phase I clinical trials $(80,81)$.

SGI-1776 is an imidazo[1,2- $\beta$ ]pyridazine small molecule inhibitor. Certain studies have reported that multiple prostate cancer, leukaemia, lymphoma and multiple myeloma cell lines treated with SGI-1776 exhibited a significant reduction in the phosphorylation levels of traditional Pim-1 substrate proteins, histone $\mathrm{H} 3$, c-Myc and Bad, interfering with proliferation and viability $(40,82-84)$. These data suggest that SGI-1776 can induce apoptosis by inhibiting Pim-1 function and producing a cytotoxic effect. SGI-1776 also has a relatively specific effect against certain paediatric cancers in vitro and in vivo with selected activated kinases at SGI-1776 concentrations, but it is more effective against AML (81). Furthermore, cells exposed to increasing doses of SGI-1776 arrested in a dose-dependent manner in the G1 cell cycle, inhibiting the natural progression to $\mathrm{S}$ phase. This was followed by apoptosis, as determined by measuring the caspase-3 activity, correlating with the downregulation of $\mathrm{p} 21^{\text {waf1 }}$ and Bad phosphorylation (82). Conversely, unlike in replicating cells, phosphorylation of traditional Pim-1 kinase targets was unaffected by SGI-1776 in CLL, indicating an alternative mechanism to induce apoptosis (85). In addition, treatment of the DU-145 prostate cancer cell line and the MiaPaCa2 pancreatic cancer cell line with SGI-1776 resulted in a significant reduction in $\mathrm{p}$-STAT3 $3^{\mathrm{Ty} 705}$ expression without affecting STAT3 expression and STAT5 phosphorylation, suggesting specificity for $\mathrm{p}-\mathrm{STAT} 3^{\mathrm{Tyr} 705}$. The inhibitory effect of SGI-1776 on STAT3 ${ }^{\text {Tyr705 }}$ phosphorylation is primarily mediated by Pim-3 in DU-145 cells (86). Siu et al (87) subsequently determined that the upregulation of MIG6 induced by SGI-1776 involved Pim-1 and that MIG6 may be a target gene of Pim-1. Recently, SGI-1776 was revealed to recover the sensitivity to doxorubicin in p-glycoprotein (ABCB1)-overexpressing cells (88). It was further identified that SGI-1776 could decrease cell surface expression of ABCB1 and the breast cancer resistance protein ABCG2 (which are substrates of Pim-1) and drug transport by Pim-1-dependent and Pim-1-independent mechanisms (89). Notably, SGI-1776 can resensitise chemoresistant cells to taxane-based therapies by inhibiting multidrug resistance activity and inducing apoptosis (82). Combination with cytarabine can increase the efficacy of Ara-C, significantly decreasing the viability of AML cell lines (90). Therefore, SGI-1776 can retard cell growth in several human haematological malignancies and solid tumours in vitro. However, phase I clinical trials have not been successful due to the cardiotoxicity of the drug. As a result, this prompted the development of antitumour drugs with more antitumour effects and fewer side effects based on the structure of this compound (91).

Quercetagetin is a type of flavonol that is also an inhibitor identified to have a moderately potent antitumour activity. 
Holder et al (92) demonstrated that quercetagetin reduces Pim-1 activity in intact RWPE2 prostate cancer cells in a dose-dependent manner to cause cell growth arrest, but it exhibited no effect on AKT kinase. The reducing effect of quercetagetin was similar to that of knockdown by siRNAs. Furthermore, the inhibitory ability of quercetagetin on cell growth was proportional to the quantity of Pim-1 protein in the target cells, particularly at lower drug concentrations. In addition, vascular SMCs markedly increased Pim-1 expression upon stimulation with PDGFbb. However, quercetagetin was able to effectively block this effect, inhibiting vascular SMC proliferation induced by PDGFbb (76). Treatment of nasopharyngeal carcinoma cell lines with quercetagetin has been demonstrated to significantly decrease cell viability, colony formation rate and migration ability via inhibition of Pim-1 overexpression (93).

The pyrrolo[2,3- $\alpha]$ carbazole has been identified as a novel scaffold on which to design potent Pim kinase inhibitors. In addition, several pyrrolo[2,3- $\alpha]$ carbazole derivatives have been identified that target Pim-1 and Pim-3 with greater selectivity than Pim-2 under in vitro conditions. The structure of this inhibitor, which has a non-ATP mimetic binding mode with no hydrogen bonds formed with the kinase hinge region, is the reason for the high selectivity of these derivatives for Pim kinases and its modest but significant selectivity for Pim-3 (94). DHPCC-9 is a potent cellular inhibitor of these derivatives, which can enter the cells and completely abrogate the anti-apoptotic effects of Pim-1 to reduce the viability of cytokine-deprived myeloid cells, whilst not exhibiting general cytotoxicity at the micromolar concentrations used. DHPCC-9 reduces all family Pim kinase activities via inhibition of the phosphorylation of their downstream substrate, Bad, whilst not reducing their endogenous expression. Moreover, DHPCC-9 removed the promigratory advantage of Pim by decreasing the motility of adherent cancer cells in a dose-dependent manner towards Pim downstream targets, such as nuclear factor of activated T-cells, cytoplasmic 1 . The reduction of cell migration in vitro by Pim-specific siRNA interference is lower than that caused by DHPCC-9, which may be due to the longer half-life and superior cell penetrance of DHPCC-9. Thus, DHPCC-9 is not only an efficient tool to research the physiological effects of the Pim family kinases, but also an attractive compound for chemotherapeutic drug development to prevent tumour metastasis or angiogenesis by inhibiting the invasiveness of cells overexpressing Pim (95).

Recently, researchers performed a virtual screening campaign that led to the identification of a series of 2-aminothiazole derivatives classified as possible allosteric inhibitors of Pim. This is a novel mechanism of inhibition that is noncompetitive with respect to ATP and the peptide substrate. Administering a combination of ATP-competitive and ATP-noncompetitive compounds highlighted a synergistic effect on the inhibition of cell proliferation in more highly metastatic cell lines, where all Pim-1 inhibitors analysed showed synergism with the known anti-cancer agent, paclitaxel. These results further establish these derivatives as promising adjuvant agents for the treatment of cancer in which Pim-1 is associated with chemotherapeutic resistance (96).

As awareness of the functions of Pim family kinases in tumourigenesis and the identification of an increasing number of novel and selective Pim kinase inhibitors has increased, the investigation and development of small molecule inhibitors targeting Pim kinases has attracted greater attention. Inhibitors of Pim kinases have been developed and synthesised; however, only certain inhibitors have been validated to have antitumour activity through cell-based assays or animal models, and only a small proportion of those can effectively inhibit the three members of the Pim family $(82,85,92,94)$. Thus, it is important to design and synthesise suitable chemotherapeutic drugs based on the three-dimensional structure of Pim kinase to inhibit its activity. Combinations of Pim inhibitors together with other chemotherapeutics may lead to more efficient therapeutic approaches.

\section{Conclusion}

Considering the gradually increasing prevalence of tumours and their high mortality rates, tumour prevention and treatment is a key area of medical research worldwide. Alternative targeting therapies, a novel direction of tumour treatment, are becoming more important as tumour incidence increases anually. The study and development of novel chemotherapeutic drugs is confronted with great opportunities and challenges. With extensive research in the field of gene therapy, the mechanisms by which Pim-1 expression is regulated are being increasingly emphasised. It is known that Pim-1 kinase, identified as an oncogene, is constitutively active and aberrantly expressed in a number of types of tumours. Additionally, Pim kinases are involved in the development of resistance against radiation therapy or chemotherapy. Functional interference with Pim-1 kinase has been recently reported to impair the growth and survival of cancer cells. As the structure and biological functions of Pim-1 are further recognised and regulators of Pim-1 expression identified, it is clear that Pim-1 has an impact on the cell cycle and apoptosis under physiological and pathological conditions. The inhibition of Pim-1 kinase expression and its activity is significant for the design and development of chemotherapeutics to treat cancer. Thus, in this review, novel strategies for tumour therapy from regulators and inhibitors of Pim-1 are discussed. Pim-1 expression is mainly regulated at the transcriptional level. However, a number of biomolecules can also mediate its expression at other levels.

MicroRNAs have emerged as a novel class of noncoding genes involved in regulating cell proliferation, differentiation and viability by knockdown of their target genes. Different cell types have different miRNA expression profiles and different stimuli can also activate the expression of different miRNAs. Identifying these stimuli and the regulatory miRNAs requires further study, which may contribute to the understanding of the complete signal pathways involving Pim-1. Moreover, an miRNA can simultaneously regulate multiple target genes, and Pim-1 is regulated by multiple miRNAs. It was hypothesised that all miRNAs could directly or indirectly regulate Pim-1 expression and subsequently regulate cell viability and survival. In the present study, certain miRNAs, which bind to the seed region of the Pim-1 3'UTR to lead to mRNA destabilisation, have been comprehensively reviewed according to the present literature. These miRNAs do not all exhibit key roles in the same types of cells and tissues. These miRNAs have been identified as tumour suppressor genes, as they can induce tumour 
cell apoptosis and inhibit tumour cell proliferation by repressing Pim-1 expression. They may be able to act as biomarkers in the research of alternative therapies targeting Pim-1. However, it remains unclear which miRNA are significantly involved in other Pim-1 overexpression tumour cells and whether the expression of miRNAs are tissue-specific. Moreover, the 3'UTR of Pim-1 harbours other miRNA binding sites, as determined by computational predictions (22). The association of miRNA regulation of Pim-1 expression and the development of relevant diseases remains unexamined.

Hormone imbalance has long been known to be relevant in the development of human tumours. The association between hormones and tumours has been further recognised in recent years, and more hormones have been revealed to be associated with Pim-1 kinase. These hormones can regulate Pim-1 via different pathways to influence tumour progression and certain biological characteristics. For example, oestrogen induces Pim-1 expression via ERBs in the promoter region, whereas DHEA decreases Pim-1 mRNA and protein levels via $\mathrm{p}$-STAT3. With increasing recognition of the functional importance of hormones targeting Pim-1 in tumourigenesis and identification of the relevant molecular mechanisms, improved choices in the treatment of hormone-dependent tumours can be developed. However, the effect of hormones is systemic and diverse, therefore pharmacological and clinical trials are required before a chemotherapeutic that targets hormones could be adopted.

A number of studies have paid increased attention to cytokines that regulate the impact of Pim-1 kinase on the tumour microenvironment (74-77,97). In this review, certain ATP mimetic inhibitors and ATP competitive inhibitors are discussed. Notably, a novel mechanism of inhibition has been recently shown to be noncompetitive with respect to ATP and the peptide substrate. Using the mechanism, inhibitors can effectively repress the activity of Pim kinases, promoting tumour cell apoptosis. Thus it is of importance for the treatment of cancer to design and develop chemotherapeutic drugs targeting Pim-1 using inhibitor scaffolds. Due to functional redundancy, simultaneous targeting of all family of Pim kinases can be advantageous in tumour therapy. However, only a few selective Pim kinase inhibitors, developed through experiments in vivo and in vitro, have exhibited antitumour activity, mainly through targeting Pim-1 and Pim-2. Moreover, certain inhibitors have not passed phase I clinical trials due to cytotoxicity. Thus, continued investigation into the crystal structure of all Pim kinases is required to identify further scaffolds inhibiting kinase activity. These results also suggest the need to consider the structure of the compound to develop antitumour drugs with more potential antitumour effects and fewer side effects. These inhibitors are still in the preliminary stages of development.

Although certain regulatory mechanisms of Pim remain unknown, the development of therapeutic agents targeting therapeutic genes in tumours in which Pim-1 is aberrantly expressed may become a novel research focus. Despite the numerous questions and obstacles that remain, it is hoped that the combined application of inhibitors of Pim-1 expression and Pim-1-specific inhibitors together with other anticancer strategies may provide novel and efficient therapies for cancer patients.

\section{Acknowledgements}

The authors would like to acknowledge grant support from the National Science Foundation of China (NSFC) (grant nos. 30973476 and 812727), the Shanghai Pujiang Programme (grant no. KW201028464), Fudan University '985 Project' Phase III Cancer Research Projects II (grant no. 985III-YFX0102) and the Shanghai Committee of Science and Technology (grant no. 12DZ2260100).

\section{References}

1. Cuypers HT, Selten G, Quint W, et al: Murine leukemia virus-induced T-cell lymphomagenesis: integration of proviruses in a distinct chromosomal region. Cell 37: 141-150, 1984.

2. Bachmann $\mathrm{M}$ and Möröy T: The serine/threonine kinase Pim-1. Int J Biochem Cell Biol 37: 726-730, 2005.

3. Mikkers H, Nawijn M, Allen J, et al: Mice deficient for all PIM kinases display reduced body size and impaired responses to hematopoietic growth factors. Mol Cell Biol 24: 6104-6115, 2004.

4. Selten G, Cuypers HT, Boelens W, et al: The primary structure of the putative oncogene pim-1 shows extensive homology with protein kinases. Cell 46: 603-611, 1986.

5. Macdonald A, Campbell DG, Toth R, McLauchlan H, Hastie CJ and Arthur JS: Pim kinases phosphorylate multiple sites on Bad and promote 14-3-3 binding and dissociation from Bcl-XL. BMC Cell Biol 7: 1, 2006.

6. Saris CJ, Domen J and Berns A: The pim-1 oncogene encodes two related protein-serine/threonine kinases by alternative initiation at AUG and CUG. EMBO J 10: 655-664, 1991.

7. Morishita D, Katayama R, Sekimizu K, Tsuruo T and Fujita N: Pim kinases promote cell cycle progression by phosphorylating and down-regulating $\mathrm{p} 27 \mathrm{Kip} 1$ at the transcriptional and posttranscriptional levels. Cancer Res 68: 5076-5085, 2008.

8. Reeves R, Spies GA, Kiefer M, Barr PJ and Power M: Primary structure of the putative human oncogene, pim-1. Gene 90: 303-307, 1990.

9. Qian KC, Wang L, Hickey ER, et al: Structural basis of constitutive activity and a unique nucleotide binding mode of human Pim-1 kinase. J Biol Chem 280: 6130-6137, 2005.

10. Nawijn MC, Alendar A and Berns A: For better or for worse: the role of Pim oncogenes in tumorigenesis. Nat Rev Cancer 11: 23-34, 2011.

11. Bullock AN, Debreczeni J, Amos AL, Knapp S and Turk BE: Structure and substrate specificity of the Pim-1 kinase. J Biol Chem 280: 41675-41682, 2005.

12. Mukaida N, Wang YY and Li YY: Roles of Pim-3, a novel survival kinase, in tumorigenesis. Cancer Sci 102: 1437-1442, 2011.

13. Mochizuki T, Kitanaka C, Noguchi K, Muramatsu T, Asai A and Kuchino Y: Physical and functional interactions between Pim-1 kinase and Cdc25A phosphatase. Implications for the Pim-1-mediated activation of the c-Myc signaling pathway. J Biol Chem 274: 18659-18666, 1999.

14. Wang Z, Bhattacharya N, Mixter PF, Wei W, Sedivy J and Magnuson NS: Phosphorylation of the cell cycle inhibitor p21Cip1/WAF1 by Pim-1 kinase. Biochim Biophys Acta 1593: 45-55, 2002.

15. Bachmann M, Hennemann H, Xing PX, Hoffmann I and Möröy T: The oncogenic serine/threonine kinase Pim-1 phosphorylates and inhibits the activity of Cdc25C-associated kinase 1 (C-TAK1): a novel role for Pim-1 at the G2/M cell cycle checkpoint. J Biol Chem 279: 48319-48328, 2004.

16. Bachmann M, Kosan C, Xing PX, Montenarh M, Hoffmann I and Möröy T: The oncogenic serine/threonine kinase Pim-1 directly phosphorylates and activates the $\mathrm{G} 2 / \mathrm{M}$ specific phosphatase Cdc25C. Int J Biochem Cell Biol 38: 430-443, 2006.

17. Nosaka T and Kitamura T: Pim-1 expression is sufficient to induce cytokine independence in murine hematopoietic cells, but is dispensable for BCR-ABL-mediated transformation. Exp Hematol 30: 697-702, 2002.

18. Brault L, Gasser C, Bracher F, Huber K, Knapp S and Schwaller J: PIM serine/threonine kinases in the pathogenesis and therapy of hematologic malignancies and solid cancers. Haematologica 95: 1004-1015, 2010. 
19. Esquela-Kerscher A and Slack FJ: Oncomirs - microRNAs with a role in cancer. Nat Rev Cancer 6: 259-269, 2006.

20. Calin GA and Croce CM: MicroRNA signatures in human cancers. Nat Rev Cancer 6: 857-866, 2006.

21. Calin GA and Croce CM: MicroRNA-cancer connection: the beginning of a new tale. Cancer Res 66: 7390-7394, 2006.

22. Thomas M, Lange-Grünweller $\mathrm{K}$, Weirauch $\mathrm{U}$, et al: The proto-oncogene Pim-1 is a target of miR-33a. Oncogene 31: 918-928, 2012.

23. Lewis BP, Burge CB and Bartel DP: Conserved seed pairing, often flanked by adenosines, indicates that thousands of human genes are microRNA targets. Cell 120: 15-20, 2005.

24. Grimson A, Farh KK, Johnston WK, Garrett-Engele P, Lim LP and Bartel DP: MicroRNA targeting specificity in mammals: determinants beyond seed pairing. Mol Cell 27: 91-105, 2007.

25. Cimmino A, Calin GA, Fabbri M, et al: miR-15 and miR-16 induce apoptosis by targeting BCL2. Proc Natl Acad Sci USA 102: 13944-13949, 2005.

26. Calin GA, Dumitru CD, Shimizu M, et al: Frequent deletions and down-regulation of micro- RNA genes miR15 and miR16 at $13 q 14$ in chronic lymphocytic leukemia. Proc Natl Acad Sci USA 99: 15524-15529, 2002.

27. Bonci D, Coppola V, Musumeci M, et al: The miR-15a-miR-16-1 cluster controls prostate cancer by targeting multiple oncogenic activities. Nat Med 14: 1271-1277, 2008.

28. Birg F, Courcoul M, Rosnet O, et al: Expression of the FMS/KIT-like gene FLT3 in human acute leukemias of the myeloid and lymphoid lineages. Blood 80: 2584-2593, 1992.

29. Nakao M, Yokota S, Iwai T, et al: Internal tandem duplication of the flt 3 gene found in acute myeloid leukemia. Leukemia 10 : 1911-1918, 1996.

30. Yokota S, Kiyoi H, Nakao M, et al: Internal tandem duplication of the FLT3 gene is preferentially seen in acute myeloid leukemia and myelodysplastic syndrome among various hematological malignancies. A study on a large series of patients and cell lines. Leukemia 11: 1605-1609, 1997.

31. Thiede C, Steudel C, Mohr B, et al: Analysis of FLT3-activating mutations in 979 patients with acute myelogenous leukemia: association with FAB subtypes and identification of subgroups with poor prognosis. Blood 99: 4326-4335, 2002

32. Kim KT, Baird K, Ahn JY, et al: Pim-1 is up-regulated by constitutively activated FLT3 and plays a role in FLT3-mediated cel survival. Blood 105: 1759-1767, 2005.

33. Kim KT, Baird K, Davis S, et al: Constitutive Fms-like tyrosine kinase 3 activation results in specific changes in gene expression in myeloid leukaemic cells. Br J Haematol 138: 603-615, 2007.

34. Kim KT, Carroll AP, Mashkani B, Cairns MJ, Small D and Scott RJ: MicroRNA-16 is down-regulated in mutated FLT3 expressing murine myeloid FDC-P1 cells and interacts with Pim-1. PLoS One 7: e44546, 2012.

35. Mizuki M, Fenski R, Halfter $\mathrm{H}$, et al: Flt3 mutations from patients with acute myeloid leukemia induce transformation of 32D cells mediated by the Ras and STAT5 pathways. Blood 96 $3907-3914,2000$

36. Tse KF, Mukherjee G and Small D: Constitutive activation of FLT3 stimulates multiple intracellular signal transducers and results in transformation. Leukemia 14: 1766-1776, 2000.

37. Hayakawa F, Towatari M, Kiyoi H, et al: Tandem-duplicated Flt 3 constitutively activates STAT5 and MAP kinase and introduces autonomous cell growth in IL-3-dependent cell lines. Oncogene 19: 624-631, 2000.

38. Nosaka T, Kawashima T, Misawa K, Ikuta K, Mui AL and Kitamura T: STAT5 as a molecular regulator of proliferation, differentiation and apoptosis in hematopoietic cells. EMBO J 18 4754-4765, 1999

39. Rayner KJ, Suárez Y, Dávalos A, et al: MiR-33 contributes to the regulation of cholesterol homeostasis. Science 328: 1570-1573, 2010

40. Tian Z, Zhao JJ, Tai YT, et al: Investigational agent MLN9708/2238 targets tumor-suppressor miR33b in MM cells. Blood 120: 3958-3967, 2012

41. Ibrahim AF, Weirauch U, Thomas M, Grünweller A, Hartmann RK and Aigner A: MicroRNA replacement therapy for miR-145 and miR-33a is efficacious in a model of colon carcinoma. Cancer Res 71: 5214-5224, 2011.

42. Chen JF, Mandel EM, Thomson JM, et al: The role of microRNA-1 and microRNA-133 in skeletal muscle proliferation and differentiation. Nat Genet 38: 228-233, 2006.

43. Zhao Y, Ransom JF, Li A, et al: Dysregulation of cardiogenesis, cardiac conduction, and cell cycle in mice lacking miRNA-1-2. Cell 129: 303-317, 2007.
44. Katakami N, Kaneto H, Hao H, et al: Role of pim-1 in smooth muscle cell proliferation. J Biol Chem 279: 54742-54749, 2004.

45. Chen J, Yin H, Jiang Y, et al: Induction of microRNA-1 by myocardin in smooth muscle cells inhibits cell proliferation. Arterioscler Thromb Vasc Biol 31: 368-375, 2011.

46. Katare RG, Caporali A, Oikawa A, Meloni M, Emanueli C and Madeddu P: Vitamin B1 analog benfotiamine prevents diabetes-induced diastolic dysfunction and heart failure through Akt/Pim-1-mediated survival pathway. Circ Heart Fail 3: 294-305, 2010.

47. Katare R, Caporali A, Zentilin L, et al: Intravenous gene therapy with PIM-1 via a cardiotropic viral vector halts the progression of diabetic cardiomyopathy through promotion of prosurvival signaling. Circ Res 108: 1238-1251, 2011.

48. Mishima T, Mizuguchi Y, Kawahigashi Y, Takizawa T and Takizawa T: RT-PCR-based analysis of microRNA (miR-1 and -124) expression in mouse CNS. Brain Res 1131: 37-43, 2007.

49. Sevignani C, Calin GA, Nnadi SC, et al: MicroRNA genes are frequently located near mouse cancer susceptibility loci. Proc Natl Acad Sci USA 104: 8017-8022, 2007.

50. Nasser MW, Datta J, Nuovo G, et al: Down-regulation of micro-RNA-1 (miR-1) in lung cancer. Suppression of tumorigenic property of lung cancer cells and their sensitization to doxorubicin-induced apoptosis by miR-1. J Biol Chem 283: 33394-33405, 2008

51. Jin Y, Tong DY, Chen JN, et al: Overexpression of osteopontin, $\alpha v \beta 3$ and Pim- 1 associated with prognostically important clinicopathologic variables in non-small cell lung cancer. PloS One 7: e 48575, 2012

52. Nieborowska-Skorska M, Hoser G, Kossev P, Wasik MA and Skorski T: Complementary functions of the antiapoptotic protein A1 and serine/threonine kinase pim-1 in the BCR/ABL-mediated leukemogenesis. Blood 99: 4531-4539, 2002.

53. Eiring AM, Harb JG, Neviani P, et al: miR-328 functions as an RNA decoy to modulate hnRNP E2 regulation of mRNA translation in leukemic blasts. Cell 140: 652-665, 2010.

54. Kulshreshtha R, Ferracin M, Wojcik SE, et al: A microRNA signature of hypoxia. Mol Cell Biol 27: 1859-1867, 2007.

55. Camps C, Buffa FM, Colella S, et al: hsa-miR-210 Is induced by hypoxia and is an independent prognostic factor in breast cancer. Clin Cancer Res 14: 1340-1348, 2008.

56. Huang X, Ding L, Bennewith KL, et al: Hypoxia-inducible mir-210 regulates normoxic gene expression involved in tumor initiation. Mol Cell 35: 856-867, 2009

57. Kelsey JL: Breast cancer epidemiology: summary and future directions. Epidemiol Rev 15: 256-263, 1993.

58. Horimoto Y, Hartman J, Millour J, et al: ER $\beta 1$ represses FOXM1 expression through targeting ER $\alpha$ to control cell proliferation in breast cancer. Am J Pathol 179: 1148-1156, 2011

59. Malinen M, Jääskeläinen T, Pelkonen M, et al: Proto-oncogene PIM-1 is a novel estrogen receptor target associating with high grade breast tumors. Mol Cell Endocrinol 365: 270-276, 2013.

60. Dumas de la Roque E, Savineau JP and Bonnet S: Dehydroepiandrosterone: A new treatment for vascular remodeling diseases including pulmonary arterial hypertension. Pharmacol Ther 126: 186-199, 2010.

61. Paulin R, Meloche J, Jacob MH, Bisserier M, Courboulin A and Bonnet S: Dehydroepiandrosterone inhibits the Src/STAT3 constitutive activation in pulmonary arterial hypertension. Am J Physiol Heart Circ Physiol 301: H1798-H1809, 2011.

62. Dhanasekaran SM, Barrette TR, Ghosh D, et al: Delineation of prognostic biomarkers in prostate cancer. Nature 412: 822-826, 2001.

63. Guzey M, Kitada S and Reed JC: Apoptosis induction by 1alpha,25-dihydroxyvitamin D3 in prostate cancer. Mol Cancer Ther 1: 667-677, 2002

64. Okamoto R, Delansorne R, Wakimoto $\mathrm{N}$, et al: Inecalcitol, an analog of $1 \alpha, 25(\mathrm{OH})(2) \mathrm{D}(3)$, induces growth arrest of androgen-dependent prostate cancer cells. Int J Cancer 130: 2464-2473, 2012

65. Ha S, Iqbal NJ, Mita P, et al: Phosphorylation of the androgen receptor by PIM1 in hormone refractory prostate cancer. Oncogene 32: 3992-4000, 2013.

66. Maier CJ, Maier RH, Rid R, et al: PIM-1 kinase interacts with the DNA binding domain of the vitamin $\mathrm{D}$ receptor: a further kinase implicated in 1,25-(OH)2D3 signaling. BMC Mol Biol 13: $18,2012$.

67. Shand RL and Gelmann EP: Molecular biology of prostate-cancer pathogenesis. Curr Opin Urol 16: 123-131, 2006. 
68. Roach M III: Current trends for the use of androgen deprivation therapy in conjunction with radiotherapy for patients with unfavorable intermediate-risk, high-risk, localized, and locally advanced prostate cancer. Cancer: Mar 3, 2014 (Epub ahead of print).

69. Carson JP, Kulik G and Weber MJ: Antiapoptotic signaling in $\mathrm{LNCaP}$ prostate cancer cells: a survival signaling pathway independent of phosphatidylinositol 3'-kinase and Akt/protein kinase B. Cancer Res 59: 1449-1453, 1999.

70. Sun A, Tang J, Hong Y, et al: Androgen receptor-dependent regulation of $\mathrm{Bcl}-\mathrm{xL}$ expression: Implication in prostate cancer progression. Prostate 68: 453-461, 2008.

71. Kumar JK, Ping RY, Teong HF, Goh S and Clément MV: Activation of a non-genomic Pim-1/Bad-Pser75 module is required for an efficient pro-survival effect of $\mathrm{Bcl}-\mathrm{xL}$ induced by androgen in LNCaP cells. Int J Biochem Cell Biol 43: 594-603, 2011.

72. Min X, Tang J, Wang Y, et al: PI3K-like kinases restrain Pim gene expression in endothelial cells. J Huazhong Univ Sci Technolog Med Sci 32: 17-23, 2012.

73. Muraski JA, Rota M, Misao Y, et al: Pim-1 regulates cardiomyocyte survival downstream of Akt. Nat Med 13: 1467-1475, 2007.

74. Zippo A, De Robertis A, Bardelli M, Galvagni F and Oliviero S: Identification of Flk-1 target genes in vasculogenesis: Pim-1 is required for endothelial and mural cell differentiation in vitro. Blood 103: 4536-4544, 2004.

75. Stout BA, Bates ME, Liu LY, Farrington NN and Bertics PJ: IL-5 and granulocyte-macrophage colony-stimulating factor activate STAT3 and STAT5 and promote Pim-1 and cyclin D3 protein expression in human eosinophils. J Immunol 173: 6409-6417, 2004.

76. Willert M, Augstein A, Poitz DM, Schmeisser A, Strasser RH and Braun-Dullaeus RC: Transcriptional regulation of Pim-1 kinase in vascular smooth muscle cells and its role for proliferation. Basic Res Cardiol 105: 267-277, 2010.

77. Block KM, Hanke NT, Maine EA and Baker AF: IL-6 stimulates STAT3 and Pim-1 kinase in pancreatic cancer cell lines. Pancreas 41: 773-781, 2012.

78. Jacobs MD, Black J, Futer O, et al: Pim-1 ligand-bound structures reveal the mechanism of serine/threonine kinase inhibition by LY294002. J Biol Chem 280: 13728-13734, 2005.

79. Gavara L, Suchaud V, Nauton L, Théry V, Anizon F and Moreau P: Identification of pyrrolo[2,3-g]indazoles as new Pim kinase inhibitors. Bioorg Med Chem Lett 23: 2298-2301, 2013.

80. Blanco-Aparicio C and Carnero A: Pim kinases in cancer: diagnostic, prognostic and treatment opportunities. Biochem Pharmacol 85: 629-643, 2013.

81. Mumenthaler SM, Ng PY, Hodge A, et al: Pharmacologic inhibition of Pim kinases alters prostate cancer cell growth and resensitizes chemoresistant cells to taxanes. Mol Cancer Ther 8: 2882-2893, 2009.
82. Chen LS, Redkar S, Taverna P, Cortes JE and Gandhi V: Mechanisms of cytotoxicity to Pim kinase inhibitor, SGI-1776, in acute myeloid leukemia. Blood 118: 693-702, 2011.

83. Yang Q, Chen LS, Neelapu SS, Miranda RN, Medeiros LJ and Gandhi V: Transcription and translation are primary targets of Pim kinase inhibitor SGI-1776 in mantle cell lymphoma. Blood 120: 3491-3500, 2012.

84. Batra V, Maris JM, Kang MH, et al: Initial testing (stage 1) of SGI-1776, a PIM1 kinase inhibitor, by the pediatric preclinical testing program. Pediatr Blood Cancer 59: 749-752, 2012.

85. Chen LS, Redkar S, Bearss D, Wierda WG and Gandhi V: Pim kinase inhibitor, SGI-1776, induces apoptosis in chronic lymphocytic leukemia cells. Blood 114: 4150-4157, 2009.

86. Chang M, Kanwar N, Feng E, et al: PIM kinase inhibitors downregulate STAT3(Tyr705) phosphorylation. Mol Cancer Ther 9: 2478-2487, 2010

87. Siu A, Virtanen C and Jongstra J: PIM kinase isoform specific regulation of MIG6 expression and EGFR signaling in prostate cancer cells. Oncotarget 2: 1134-1144, 2011.

88. Xie Y,Burcu M,Linn DE, Qiu Y and Baer MR: Pim-1 kinase protects P-glycoprotein from degradation and enables its glycosylation and cell surface expression. Mol Pharmacol 78: 310-318, 2010.

89. Natarajan K, Bhullar J, Shukla S, et al: The Pim kinase inhibitor SGI-1776 decreases cell surface expression of P-glycoprotein (ABCB1) and breast cancer resistance protein (ABCG2) and drug transport by Pim-1-dependent and -independent mechanisms. Biochem Pharmacol 85: 514-524, 2013.

90. Kelly KR, Espitia CM, Taverna P, et al: Targeting PIM kinase activity significantly augments the efficacy of cytarabine. Br J Haematol 156: 129-132, 2012.

91. Pogacic V, Bullock AN, Fedorov O, et al: Structural analysis identifies imidazo[1,2-b]pyridazines as PIM kinase inhibitors with in vitro antileukemic activity. Cancer Res 67: 6916-6924, 2007.

92. Holder S, Zemskova M, Zhang C, et al: Characterization of a potent and selective small-molecule inhibitor of the PIM1 kinase. Mol Cancer Ther 6: 163-172, 2007.

93. Jie W, He QY, Luo BT, et al: Inhibition of Pim-1 attenuates the proliferation and migration in nasopharyngeal carcinoma cells. Asian Pac J Trop Med 5: 645-650, 2012.

94. Akué-Gédu R, Rossignol E, Azzaro S, et al: Synthesis, kinase inhibitory potencies, and in vitro antiproliferative evaluation of new Pim kinase inhibitors. J Med Chem 52: 6369-6381, 2009.

95. Santio NM, Vahakoski RL, Rainio EM, et al: Pim-selective inhibitor DHPCC-9 reveals Pim kinases as potent stimulators of cancer cell migration and invasion. Mol Cancer 9: 279, 2010.

96. Mori M, Tintori C, Christopher RS, et al: A combination strategy to inhibit Pim-1: synergism between noncompetitive and ATP-competitive inhibitors. ChemMedChem 8: 484-496, 2013.

97. Grundler R, Brault L, Gasser C, et al: Dissection of PIM serine/threonine kinases in FLT3-ITD-induced leukemogenesis reveals PIM1 as regulator of CXCL12-CXCR4-mediated homing and migration. J Exp Med 206: 1957-1970, 2009. 